\title{
Astrocitomas Difusos de Baixo Grau de Malignidade
}

\author{
Suzana Maria Fleury Malheiros*, João Norberto Stávale**, Clélia \\ Maria Ribeiro Franco**, Fernando Menezes Braga***, Alberto \\ Alain Gabbai*****
}

\section{RESUMO}

Os autores apresentam uma revisão sobre astrocitomas de baixo grau de malignidade (Grau II - OMS), ressaltando, particularmente, os aspectos controversos em relação ao tratamento desses tumores.

\section{UNITERMOS}

Astrocitoma, glioma, radioterapia.
* Chefe do Setor de Neuro-oncologia da Escola Paulista de Medicina - UNIFESP.

** Prof. Adjunto da Disciplina de Patologia Cirúrgica da Escola Paulista de Medicina UNIFESP.

*** Pós-graduanda da Disciplina de Neurologia da Escola Paulista de Medicina - UNIFESP.

**** Prof. Titular da Disciplina de Neurocirurgia da Escola Paulista de Medicina - UNIFESP.

${ }^{* * * \star *}$ Prof. Titular da Disciplina de Neurologia da Escola Paulista de Medicina - UNIFESP.
Os chamados "gliomas de baixo grau" correspondem de 10 a $20 \%$ dos tumores primários do $\mathrm{SNC}$ em adultos ${ }^{20}$. Esse grupo inclui astrocitomas (mais freqüentes), oligodendrogliomas, gliomas mistos (oligoastrocitomas), além de variantes menos comuns de astrocitomas, como o astrocitoma pilocítico, o xantoastrocitma pleomórfico e o astrocitoma subependimário de células gigantes ${ }^{28}$. São tumores que acometem com mais freqüência crianças e adultos jovens ${ }^{14}$, com idade mediana de 37,3 anos na época da apresentação (variação em casos extremos de 7 meses a 78 anos $)^{35}$, e predomínio no sexo masculino ${ }^{15}$.

Embora englobados na classificação como "gliomas de baixo grau", sabese que os astrocitomas, oligodendrogliomas e oligoastrocitomas apresentam características clínicas, comportamento biológico, resposta a tratamento e prognóstico diferentes. Mesmo entre o grupo específico dos astrocitomas, a inclusão de tumores com comportamento biológico e prognósticos bastante diversos, como o astrocitoma pilocítico ${ }^{15}$, xantoastrocitma pleomórfico e astrocitoma subependimário de células gigantes ${ }^{23}$, torna esse grupo muito heterogêneo. Portanto, nesta revisão optamos por discutir, especificamente, as características dos astrocitomas difusos grau II (Organização Mundial da Saúde - OMS) e as grandes controvérsias que envolvem a conduta nos pacientes com este diagnóstico.

\section{PATOLOGIA}

Os astrocitomas difusos de baixo grau de malignidade são também chamados simplesmente de astrocitomas ${ }^{14}$, e correspondem aos chamados astrocitomas grau II pela $\mathrm{OMS}^{14}$, ou astrocitomas grau I e II pela classificação de St. Anne-Mayo ${ }^{7}$. São tumores celulares, heterogêneos, geralmente pouco delimitados e infiltrativos, que apresentam pleomorfismo nuclear, sem sinais de anaplasia (mitoses, proliferação endotelial e necrose) e podem ocorrer em qualquer parte do $\mathrm{SNC}^{15}$.

$\mathrm{O}$ aumento de celularidade pode ser discreto, simulando um processo de gliose reacional, o que pode dificultar o diagnóstico do patologista: a presença 
de microcistos, citoplasma escasso e cromatina mais espessa são características úteis para o diagnóstico diferencial ${ }^{3,15}$. Podem ser encontradas microcalcificações em $15 \%$ dos casos $^{3}$.

Os astrocitomas grau II originam-se a partir de precursores - astrócitos tipo 1 ou células da linhagem O2A. Provavelmente mutações do p53 e perda da heterozigose do cromossomo $17 \mathrm{p}$ sejam passos importantes para a gênese desses tumores ${ }^{20}$.

Astrócitos protoplasmáticos são encontrados com freqüência no córtex e núcleos da base, enquanto os astrócitos fibrilares são mais encontrados na substância branca, próximos a estruturas vasculares. A distinção entre astrocitomas fibrilares (mais freqüentes) e os protoplasmáticos é feita com base na presença de fibrilas neurogliais, associadas com os astrocitomas fibrilares. Ambos são difusos e infiltrativos, expressando proteína glial fibrilar acídica (GFAP) na imunohistoquímica ${ }^{3}$.

Astrocitomas gemistocíticos são mais raros e mais corticais. Contêm células com material hialino responsável por sua aparência globosa. Embora considerados como astrocitomas grau II, esses tumores podem comportar-se de forma mais agressiva, com maior propensão a evoluir para tumores anaplásicos (astrocitoma anaplásico e glioblastoma multiforme) ${ }^{14,16,20,28}$.

Apesar de as características histológicas sugerirem crescimento lento e longa sobrevida, os astrocitomas grau II não devem ser chamados de "benignos" devido à sua localização, natureza infiltrativa, morbidade e risco de evolução para graus mais malignos ${ }^{3,14,15,20,34,35}$. Na maioria das vezes, eles serão os responsáveis pela morte dos pacientes afetados ${ }^{3,18}$.

\section{QUADRO CLÍNICO}

Como em qualquer tumor do SNC, a apresentação clínica depende da localização: crises epilépticas, cefaléia, sinais de hipertensão intracraniana, decorrente do efeito expansivo ou de hidrocefalia, e déficits focais são os sintomas mais freqüentes. A forma de apresentação dos sintomas costuma ser lenta e insidiosa.

\section{DIAGNÓSTICO POR EXAMES DE IMAGEM}

A maioria dos astrocitomas grau II apresenta-se como lesão única hipoatenuante na tomografia de crânio, em localização lobar (especialmente frontal e temporal), geralmente pouco delimitada e sem realce após injeção de contraste ${ }^{35}$. Na ressonância magnética (RM), a qual fornece mais detalhes anatômicos e melhor definição da extensão do tumor, costumam apresentar hipossinal em T1 e hipersinal em T2 e DP, muitas vezes com aspecto infiltrativo e geralmente sem realce.

Embora a presença de realce após contraste indique, na maioria das vezes, a possibilidade de tumores anaplásicos, cerca de $20 \%$ dos astrocitomas de baixo grau podem apresentar realces ${ }^{25,27}$. É importante ressaltar, por outro lado, a existência de astrocitomas anaplásicos apresentarem aspecto semelhante aos astrocitomas grau II nos exames de imagem, não realçando após contraste ${ }^{1,5,11,17}$

Raramente, pode haver dificuldade em se diferenciar o quadro de gliose, encefalite, doença desmielinizante ou quadro vascular, especialmente na tomografia de crânio, sendo, nestes casos, necessária a confirmação anatomopatológica para o diagnóstico.

\section{TRATAMENTO}

A conduta nos astrocitomas grau II é ainda um dos assuntos mais controversos em neuro-oncologia. Os tratamentos disponíveis não são curativos, embora possam proporcionar melhora dos sintomas e da qualidade de vida, e visam prolongar a sobrevida, na esperança de tratamentos mais eficazes no futuro.

Não há grandes estudos prospectivos controlados para avaliar o efeito das diferentes modalidades de tratamento desses tumores e, obviamente, as condutas baseadas em estudos retrospectivos são sempre sujeitas a muitas críticas ${ }^{26}$, como, por exemplo, falhas na seleção dos pacientes, inclusão de diferentes tipos histológicos com prognósticos diferentes, necessidade de longo tempo de acompanhamento e de controle de muitas variáveis que interferem no prognóstico, etc. Por esse motivo, os estudos retrospectivos devem ser interpretados com cuidado ${ }^{4}$.

\section{Cirurgia}

O papel da cirurgia no tratamento nunca foi objeto de um estudo randomizado controlado, entretanto, a maioria dos neuro-oncologistas é favorável à ressecção mais ampla possível. Os progressos recentes na neurocirurgia - estereotaxia, ultra-som, eletrocorticografia etc. - têm contribuído para a segurança e sucesso do tratamento cirúrgico.

A proposta de cirurgia nos astrocitomas grau II tem vários objetivos, apesar das controvérsias: 
1. Confirmação do diagnóstico: afastar, por exemplo, AVC, encefalites ou doenças desmielinizantes, nos casos duvidosos.

2. Diagnóstico do tipo histológico do tumor: essencial para afastar a possibilidade de gliomas anaplásicos que não realçam, além de estabelecer o diagnóstico histológico específico do tipo de glioma, o que influi no tratamento e prognóstico. No caso de oligodendrogliomas e gliomas mistos, por exemplo, pode haver melhor resposta à quimioterapia ${ }^{20}$, e a sobrevida nesses pacientes costuma ser maior do que nos pacientes com astrocitoma ${ }^{28}$.

3. Ressecção de maior volume possível do tumor, desde que segura, pode contribuir para melhorar o quadro clínico, permitindo, por exemplo, o controle de crises e hipertensão intracraniana. Por outro lado, apesar de os astrocitomas serem geralmente infiltrativos, não passíveis de cura pela cirurgia e, com freqüência, recidivarem nas margens, mesmo após ressecção completa, muitos autores consideram a ressecção completa como um fator prognóstico favorável. A indicação precoce da cirurgia baseia-se na hipótese, ainda não confirmada em estudos prospectivos, de que a ressecção de tumores menores possa reduzir a recorrência e a transformação maligna ${ }^{35}$.

Alguns autores, entretanto, defendem a conduta expectante nos pacientes com tumores pequenos, com crises controladas. Nesse caso, sugerem que os pacientes poderiam ser acompanhados, clinicamente e através de exames de imagem, até que houvesse progressão, caracterizada pela piora clínica ou crescimento do tumor, ou ainda aparecimento de realce $^{4,28}$. Segundo esses autores, seria possível evitar, dessa forma, os efeitos deletérios potenciais do tratamento cirúrgico em pacientes com sobrevida que pode ser relativamente longa, considerando-se o comportamento biológico do tumor, muitas vezes indolente ${ }^{4}$. As maiores críticas a esse tipo de conduta estão no fato de que os exames de imagem (CT e RM) ainda não sejam capazes de diagnosticar o tipo histológico do tumor, o que determina a escolha do tratamento específico. Astrocitomas anaplásicos, oligodendrogliomas e oligoastrocitomas, como já comentado, podem ter o mesmo aspecto de ausência de realce nos exames de imagem, e seu tratamento específico difere daquele do astrocitoma. Por outro lado, astrocitomas pilocíticos e xantoastrocitomas pleomórficos (que apresentam um prognóstico mais favorável) e alguns astrocitomas grau II podem apresentar realce nos exames de imagem após contraste, sendo, erroneamente, confundidos com gliomas anaplásicos de pior prognóstico. Além disso, a conduta expectante exige acompanhamento rigoroso do paciente ${ }^{20}$, o que nem sempre é possível em nosso meio (pacientes de outras cidades e estados, dificuldades em se obter exames de imagem etc.).

A biópsia tem sido reservada a pacientes com tumores inacessíveis ao tratamento cirúrgico: a possibilidade de erros de amostragem deve ser sempre lembrada, e a obtenção de várias amostras de tecido de locais diferentes do tumor é sempre recomendada.

\section{TRATAMENTO SUPLEME NTAR}

O tratamento dos astrocitomas de baixo grau após a cirurgia depende, basicamente, da histologia, do grau de ressecção, da idade e do quadro clínico, bem como da expectativa do paciente e de sua família.

\section{Radioterapia}

O uso da radioterapia no tratamento dos astrocitomas grau II é outro assunto extremamente controverso, em especial nos pacientes com ressecção cirúrgica completa.

Vários autores demonstraram, em estudos retrospectivos, uma vantagem na sobrevida dos pacientes submetidos a radioterapia, em geral com doses de aproximadamente $5500 \mathrm{cGy}^{10,18,19,22,24,34}$. Não há grandes estudos prospectivos controlados para avaliar o efeito da radioterapia no tratamento desses tumores.

Considerando-se que a maioria dos astrocitomas difusos e gemistocíticos apresenta risco de recidiva precoce, a radioterapia tem sido sugerida como tratamento adjuvante por vários autores ${ }^{9,19}$. Nesses casos, recomenda-se a radioterapia convencional localizada e fracionada, na dose de 5400 a 6000 cGy. A identificação de células tumorais a vários centímetros de distância das margens da alteração de sinal, nas imagens de RM em T2 ${ }^{13}$, sugere que o tratamento local deva incluir uma margem de 2 a $3 \mathrm{~cm}$ além da alteração de sinal em T2.

É importante ressaltar que a radioterapia não é isenta de efeitos colaterais. Embora o atual desenvolvimento técnico dos métodos de imagem e da própria radioterapia permitam o tratamento cada vez mais localizado, devemos lembrar que a sobrevida relativamente longa dos pacientes com astrocitomas grau II pode permitir o aparecimento de efeitos tardios deletérios da radioterapia ${ }^{6,12,21,31}$, como por exemplo, radionecrose, demência e alterações cognitivas, alterações endocrinológicas e perdas visuais, além de tumores secundários, fato que não se observa nos gliomas anaplásicos, por sua menor sobrevida. Embora vários autores ${ }^{30,38}$ 
concordem que a radioterapia possa retardar ou prevenir o risco de transformação maligna do tumor, o risco de contribuir para acelerar essa evolução já foi aventado ${ }^{4,20}$, embora não comprovado.

$\mathrm{Shaw}^{33}$ ressalta a raridade de complicações mais graves da radioterapia, como radionecrose $(2 \%)$ e tumores secundários, embora devamos considerar que esse risco possa estar sendo subestimado, uma vez que nem sempre os pacientes são submetidos a biópsias confirmatórias. Baseado nessa afirmação, este autor propõe que não se deve discutir a indicação da radioterapia, mas sim a dose a ser utilizada.

Talvez a questão a ser definida não seja tratar ou não esses pacientes, mas sim a época ideal do tratamento: no momento do diagnóstico ou na progressão? ${ }^{20,32}$

Alguns estudos prospectivos em andamento procuram responder a essas questões: a indicação de radioterapia precoce versus tardia, diferentes esquemas e doses etc ${ }^{20}$.

As vantagens do uso de outras modalidades de radioterapia localizada como braquiterapia e radiocirurgia ainda não estão definidas e também têm sido avaliadas em estudos prospectivos em andamento ${ }^{35}$. A grande ressalva, nesses casos, é o maior risco de radionecrose nessas formas de radioterapia.

\section{Quimi oterapia (QT)}

A maioria dos tumores da linhagem astrocítica não responde à quimioterapia, especialmente no caso dos tumores de baixo grau de malignidade ${ }^{20}$. Em alguns casos de tumores mais agressivos e, especialmente, nas crianças, nas quais se procura evitar a radioterapia por seus efeitos potencialmente deletérios a longo prazo, vários esquemas de QT têm sido tentados, sem sucesso até o momento. Eyre et al. ${ }^{8}$, em um estudo randomizado, não encontraram benefício da QT adjuvante com CCNU em pacientes com gliomas de baixo grau com ressecção parcial.

\section{TRATAME NTO NA RECORRÊNCIA}

$\mathrm{Na}$ recorrência, todos os pacientes devem ser avaliados quanto à possibilidade de reoperação ou biópsia (afastar radionecrose), especialmente quando houver acesso cirúrgico e nos pacientes com bom índice de Karnofsky. Braquiterapia e radiocirurgia podem ser boas opções nessas situações ${ }^{20}$. A quimioterapia pode, também, apresentar alguma resposta, especialmente nas transformações malignas ${ }^{39}$.

\section{PROGNÓSTICO}

Embora os astrocitomas grau II tenham características histológicas não agressivas, não podem ser considerados como tumores benignos, uma vez que a maioria dos pacientes morre em conseqüência deles, que geralmente evoluem ou recidivam de forma mais agressiva. A mediana de sobrevida esperada para pacientes com astrocitomas difusos é de 5 a $8 \operatorname{anos}^{28}$, enquanto pacientes com astrocitomas gemistocíticos tendem a evoluir com menor sobrevida ${ }^{15}$.

Alguns fatores parecem influir no prognóstico:

1. Tipo histológico: provavelmente o fator prognóstico mais importante ${ }^{15,18,19,34}$.

2. Idade: vários estudos retrospectivos demonstraram maior sobrevida mediana em pacientes mais jovens $(<40 \text { anos })^{8,18,22,34}$. Vecht ${ }^{37}$, em uma revisão sobre o assunto, chega a considerar a idade como fator prognóstico independente e sugere que decisões quanto ao tratamento, mais ou menos agressivo, sejam baseadas na idade. Devemos lembrar, porém, que ele baseia sua opinião em estudos retrospectivos.

3. Extensão da ressecção: ressecções mais amplas têm sido consideradas como um fator de bom prognóstico, quando comparadas com biópsias ou ressecções parciais ${ }^{2,8,18,30,36}$.

4. Potencial proliferativo: o índice de proliferação celular vem sendo estudado, nos últimos anos, como forma de tentar prever o comportamento dos tumores, e talvez possa indicar os pacientes que devam ser tratados mais agressivamente ${ }^{20,35}$. A bromodeoxiuridina (BUdR) necessita ser administrada antes da cirurgia, impossibilitando seu uso em estudos retrospectivos, porém, o PCNA e o antígeno Ki-67 podem ser usados em amostras de tecido, sendo portanto mais úteis na prática diária ${ }^{20}$.

- BUdR e antígeno nuclear de células em proliferação (PCNA) medem a porcentagem de células em fase $S$;

- Citometria de fluxo mede a porcentagem de células em fase $\mathrm{S}$ e G2M;

- Anticorpo monoclonal Ki-67 mede a porcentagem de células em fase $\mathrm{G} 1+\mathrm{S}+\mathrm{G} 2+\mathrm{M}$;

4. O estado funcional do paciente ${ }^{8,20}$, baseado no índice de Karnofsky pós-operatório, reflete indiretamente a localização e extensão do tumor.

5. Outros fatores são também considerados importantes, embora não universalmente aceitos ${ }^{20}$, como longa duração dos sintomas, crises como primeiro sintoma, ausência de déficit neurológico e tumores com componentes císticos. 
Embora inúmeras questões permaneçam sem respostas, em resumo, algumas tendências podem ser sugeridas no tratamento dos astrocitomas grau II:

1. Tumores acessíveis ou sintomáticos devem ser operados com ressecção mais ampla possível, dependendo, evidentemente, da qualidade e experiência do serviço de neurocirurgia.

2. Pacientes com ressecção completa provavelmente podem ser observados cuidadosamente, a princípio, aguardando para iniciar a radioterapia em caso de progressão. A radioterapia deve ser iniciada imediatamente nos pacientes acima de 35 anos?

3. Pacientes com ressecção parcial podem ser observados de início, ou devem iniciar radioterapia imediatamente? Iniciar radioterapia apenas nos pacientes acima de 35 anos?

4. Pacientes com tumores pequenos, assintomáticos (exceto pelas crises controladas), em zonas eloqüentes, podem ser observados cuidadosamente até a progressão clínica ou de imagem?

5. Pacientes com tumores sintomáticos, em progressão, e irressecáveis devem ser submetidos a biópsia (apesar do risco de erro de amostragem) ou ressecção parcial e radioterapia complementar, caso se confirme o diagnóstico de astrocitoma grau II.

6. Em todas as situações acima é importante ressaltar a necessidade de acompanhamento rigoroso dos pacientes.

7. Exceto em situação de protocolos de pesquisa, a decisão deve ser feita com base em cada caso, considerando-se também a opinião do paciente e de seus familiares.

8. Em todas as situações citadas nos itens 2 a 4, resta saber como prever quais desses tumores terão evolução mais indolente (índices de proliferação celular?), como determinar qual o melhor intervalo para o seguimento do paciente, e quais pacientes seguirão rigorosamente as orientações médicas.

Estudos prospectivos em andamento, provavelmente, contribuirão para esclarecer essas questões.

\section{SUMMARY}

Low-grade Astrocytomas

The authors review the main controversies about the management of lowgrade astrocytoma.

\section{KEY WORDS}

Astrocytoma, glioma, radiotherapy.

\section{Referências}

1. Barker, F.G.; Chang, S.M.; Huhn, S.L.; Davis, R.L. et al. Age and the risk of anaplasia in magnetic resonance-nonenhancing supratentorial cerebral tumors. Cancer, 80: 936-41, 1997.
2. Berger, M.S.; Deliganis, A.V.; Dobbins, J.; Keles, G.E. The effect of extent of resection on recurrence in patients with low-grade cerebral hemisphere gliomas. Cancer, 74: 17841791, 1994

3. Bruner, J.M. Neuropathology of malignant gliomas. Sem Oncol, 21: 126-138, 1994.

4. Cairncross, J.G.; Laperriere, N.J. Low-grade glioma. To treat or not to treat? Arch Neurol, 46: 1238-1239, 1989.

5. Chamberlain, M.C.; Murovic, J.A.; Levin V.A. Absence of contrast enhancement on CT brain scans of patients with supratentorial malignant gliomas. Neurology, 38: 1371-1374, 1988.

6. Constine, L.S.; Woolf, P.D.; Cann, D.; Mick, G. et al. Hypothalamic-pituitary dysfunction after radiation for brain tumors. N Engl J Med, 328: 87-94, 1993.

7. Daumas-Duport, D.; Scheithauer, B.; O’Fallon, J.; Kelly, P. Grading of astrocytomas. A simple and reproducible method. Cancer, 62: 2152-2165, 1988.

8. Eyre, H.J.; Crowley, J.J.; Townsend, J.J.; Eltringham, J.R. et al. A randomized trial of radiotherapy versus radiotherapy plus CCNU for incompletely resected low-grade gliomas: a Southwest Oncology Group study. J. Neurosurg, 78: 909-914, 1993.

9. Fazekas, J.T. Treatment of grades I and II brain astrocytomas. The role of radiotherapy. Int J Radiat Oncol Biol Phys, 2: 661. 666, 1977.

10. Garcia, D.M.; Fulling, K.H.; Marks, J.E. The value of radiation therapy in addition to surgery for astrocytomas of the adult cerebrum. Cancer, 55: 919-927, 1985.

11. Gruber, M.; Hochberg, F. Systematic evaluations of primary brain tumors. J Nucl Med, 31: 969-970, 1987.

12. Hochberg, F.H.; Slotnick, B. Neuropsychologic impairment in astrocytomas survivors. Neurology, 30: 172-177, 1980.

13. Kelly, P.J.; Daumas-Duport, C.; Kispert, D.B.; Kall, B.A. et al. Imaging-based stereotaxic serial biopsies in untreated intracranial glial neoplasms. J Neurosurg, 66: 865-874, 1987.

14. Kleihues, P.; Burger, P.C.; Scheithauer B.W. (eds.) Histological typing of tumours of the Central Nervous System, Berlin: Springer-Verlag, 1993.

15. Kleihues, P.; Davis, R.L.; Ohgaki, H.; Cavanee, W.K. Low-grade diffuse astrocitomas. In: Kleihues, P.; Cavanee, W.K. (eds.) Pathology and genetics of tumours of the nervous system, Lyon: International Agency for Research on Cancer, 1997, p. 10-14.

16. Krouwer, H.G.J.; Davis, R.L.; Silver, P.; Prados, M. Gemistocitic astrocytomas: a reappraisal. J Neurosurg, 74: 399-406, 1991.

17. Lassoff, S.J.; Hochberg, F.H.; Skates, S.J. Low-grade gliomas: assumption in the evaluation of low-absorption CT masses. Neurology, 39(suppl 1): 227, 1989.

18. Laws, E.R.; Taylor, W.F.; Clifton, M.B.; Okaziki, H. Neurosurgical management of low-grade astrocytoma of cerebral hemispheres. J Neurosurg, 61: 665-673, 1984.

19. Leibel, S.A.; Sheline, G.E.;Wara, W.M.; Boldrey, E.B.; Nielsen, S.L. The role of radiation therapy in the treatment of astrocytomas. Cancer, 35: 1551-1557, 1975.

20. MacDonald, D.R. Low-grade gliomas, mixed gliomas, and oligodendrogliomas. Sem Oncol, 21: 236-248, 1994.

21. Marie, J.P.; Coudin, B.; Guerin, J.; Caudry, M. Neuropsychologic impairment in adults with brain tumors. Am J Clin Oncol, 10: 156-162, 1987

22. McCormack, B.M.; Miller, D.C.;Budzilovich, G.N.;Voorhees, G.J.; Ranshohoff, J. Treatment and survival of low-grade astrocytoma in adults: 1977-1988. Neurosurgery, 31: 636-642, 1992.

23. Ng, H.K.; Leung, S.Y. The new WHO classification of CNS tumors: a selective review. Adv Anat Pathol, 2: 195-207, 1995.

24. North, C.A.; North, R.B.; Epstein, J.A.; Piantadosi, S.; Wharan, M.D. Low-grade astrocytomas. Survival and quality of life after radiation therapy. Cancer, 66: 6-14, 1990. 
25. Pedersen, H.; Gjerris, F.; Kliken, L. Malignancy criteria in computed tomography of primary supratentorial tumors in infancy and childhood. J Neurosurg, 68: 698-704, 1989.

26. Perry, J.R.; DeAngelis, L.M.; Schold, S.C.; Burger, P.C. et al. Challenges in the design and conduct of phase III brain tumor therapy trials. Neurology, 49: 912-917, 1997.

27. Piepmeier, J.M. Observations on the current treatment of lowgrade astrocytic tumors of the cerebral hemispheres. J Neurosurg, 67: 177-181, 1987.

28. Recht, L.D.; Bernstein, M. Low-grade gliomas. In: Wen, P.Y; Black, P.M.; eds: Neurologic Clinics. Brain tumors in adult, Philadelphia: W. B. Saunders Company, 1995, p. 847-859.

29. Recht, L.D.; Lew, R.; Smith, T.W. Suspected low-grade glioma: is deferring treatment safe? Ann Neurol, 31: 431-436, 1992.

30. Reichenthal, E.; Feldman, Z.; Cohen, M.L.; Loven, D.; Zucker, G. Hemispheric supratentorial low-grade gliomas. Neurochirurgia, 35: 18-22, 1992.

31. Shapiro, W.R. Treatment of neuroectodermal brain tumors. Ann Neurol, 12: 231-237, 1982.

32. Shapiro, W.R. Low-grade gliomas: When to treat? Ann Neurol, 31: 437-438, 1992.

33. Shaw, E.G. Low-grade gliomas: to treat or not to treat? A radiation oncologist's viewpoint. Arch Neurol, 47: 1138-1139, 1990.

34. Shaw, E.G.; Daumas-Duport, C.; Scheithauer, B.W.; Gilbertson, D.T. et al. Radiation therapy in the management of supratentorial astrocitomas. J Neurosurg, 70: 853-61, 1989.
35. Shaw, E.G.; Scheithauer, B.W.; Dinapoli, R.P. Low-grade hemispheric astrocytomas. In: Black, P.M.; Loeffler, J.S. (eds.) Cancer of the nervous system. Cambridge: Blackwell Science, 1997, p. 441-463.

36. Soffietti, R.; Chio, A.; Giordana, M.T.; Vasario, E.; Schiffer, D. Prognostic factors in well differentiated cerebral astrocytomas. Neurosurgery, 24: 686-692, 1989.

37. Vecht, C.T. Effect of age on treatment decisions in low-grade glioma. J Neurol Neurosurg Psy, 56: 1259-1264, 1993.

38. Vertosick Jr., F.T.; Selker, R.G.; Arena, V.C. Survival of patients with well-differentiated astrocytomas diagnosed in the era of computed tomography. Neurosurgery, 28: 496-501, 1991.

39. Wringer, M.J.; MacDonald, D.R.; Cairncross, J.G. Supretentorial anaplastic gliomas in adults. The prognostic importance of extent of resection and prior low-grade glioma. J Neurosurg, 71: 487-493, 1989.

\section{Endereço para correspondência:}

Suzana M. F. Malheiros

Disciplina de Neurologia

Rua Botucatu, 740

CEP 04023-900 São Paulo (SP)

e-mail: suzana@sun-nepi.epm.br 